\title{
APLICAÇÃO DA TEORIA CAÓTICA DETERMINISTA NO ESTUDO DA CONCENTRAÇÃO ATMOSFÉRICA DE MATERIAL PARTICULADO INALÁVEL E MONÓXIDO DE CARBONO
}

\author{
W. H. PRIETO ${ }^{*}$, E. TOMAZ ${ }^{1}$, M. A. CREMASCO ${ }^{1}$ \\ ${ }^{1}$ Universidade Estadual de Campinas, Faculdade de Engenharia Química, Departamento de \\ Engenharia de Processos \\ *e-mail: wesley@feq.unicamp.br
}

\begin{abstract}
RESUMO
A complexa dinâmica envolvida na poluição atmosférica promove a necessidade de metodologias de análise que representem, com maior fidelidade, suas características físicas. Nas últimas décadas, diversas pesquisas demonstraram os efeitos nocivos dos contaminantes atmosféricos à saúde humana, fauna e flora, desta forma explicitando a urgência do conhecimento das causas, padrões e mecanismos deste fenômeno. Os métodos clássicos empregados para este fim, frequentemente, negligenciam as interações não lineares presentes na evolução temporal dos poluentes. Nesta vanguarda, o presente trabalho aplica a teoria caótica determinista no estudo de séries temporais de concentração de material particulado inalável $\left(\mathrm{PM}_{10}\right)$ e monóxido de carbono fornecidas pela estação de monitoramento do Parque do Ibirapuera (São Paulo) pertencente à Companhia Ambiental do Estado de São Paulo (CETESB). Foram obtidos os atratores no espaço de fases e, a partir de sua topografia, determinou-se os valores dos invariantes dimensão de correlação $\left(\mathrm{D}_{2}\right)$ e entropia de Kolmogorov $(\mathrm{K})$. Com base nessas grandezas, observou-se a natureza não linear, aperiódica e determinística dos dados avaliados, demonstrando que a teoria do caos é uma alternativa viável no estudo de poluição atmosférica.
\end{abstract}

\section{INTRODUÇÃ̃O}

A relação entre o aumento da concentração dos poluentes atmosféricos e a incidência de diversos problemas de saúde da população é evidente (MARTIN e BRADLEY, 1960; SALDIVA et al., 1995; DUCHIADE, 1992; BRAGA, 1999), sendo a poluição um grave problema de saúde pública. Nas metrópoles, a frota de veículos automotores e as crescentes emissões de poluentes tóxicos pelas chaminés das indústrias, conforme mencionado por Derisio (1992), provocam altas concentrações de substâncias nocivas que são responsáveis pela baixa visibilidade e diversos problemas respiratórios nos seres vivos (AMDUR et al., 1991; DOCKERY et al., 1993; POPE et al., 2002). Com o advento da Revolução Industrial pouco se fez para controlar ou estudar esses efeitos, sendo os episódios de inversão térmica, relacionados a eventos meteorológicos, os responsáveis por impulsionar a comunidade científica a averiguar, certificar e relacionar os índices de mortalidade em centros urbanos à poluição atmosférica (DOCKERY e POPE, 1994).

As concentrações de particulados inaláveis $\left(\mathrm{PM}_{10}\right)$ e monóxido de carbono $(\mathrm{CO})$ merecem especial atenção neste cenário. $\mathrm{O}$ CO é um gás altamente tóxico cujas emissões são, predominantemente, oriundas de veículos 
automotores. Em relação aos particulados inaláveis, os efeitos de seus níveis atmosféricos podem ocasionar desde tosse seca, cansaço, ardor nos olhos, nariz e garganta até sérios riscos de manifestação de doenças respiratórias e cardiovasculares e aumento das mortes prematuras em pessoas de grupos sensíveis (crianças, idosos e acometidos de doenças do aparelho respiratório e cardíaco) (CETESB, 2014).

Diante da urgência do controle da emissão de poluentes, agências governamentais e privadas dispendem recursos para financiar pesquisas para quantificar e controlar as concentrações desses compostos, gerando assim indicadores de qualidade do ar. Neste cenário, lança-se mão de metodologias que, muitas vezes, consideram como lineares as interações entre os poluentes atmosféricos (HELLEBUST et al., 2010; GUO, 2011), assim, negligenciando o caráter não linear da evolução deste fenômeno. Muitos autores aplicam a análise fatorial dinâmica (DFA) objetivando analisar características estocásticas por meio da decomposição de uma série temporal multivariada em combinações lineares dos fatores majoritários e suas variações temporais comuns associadas (YU et al., 2013), porém esta metodologia não possibilita uma avaliação fidedigna de todas as, já solidificadas, relações dinâmicas não lineares presentes nos sistemas atmosféricos (LORENZ, 1963; TSONIS, 1992). A aplicação de técnicas não lineares como a análise no domínio do tempo, no domínio da frequência ou no domínio do espaço (análise de caos) (JOHNSSON et al., 2000) são, também, consideradas como alternativas na análise da dispersão de poluentes. Um dos métodos consagrados para o estudo destas séries temporais é a análise espectral (domínio da frequência) (HONG et al., 1990; BAI et al., 1999; JOHNSSON et al., 2000; KAGE et al., 1991; TRNKA et al., 2000; BROWN e BRUE, 2001; SASIC et al., 2007; MARTÍN et al., 2011). Esta metodologia normalmente utiliza a transformada de Fourier e visa a obtenção de frequências dominantes, características a cada fenômeno, presentes no espectro de potências (KAGE et al., 1991). No que tange esta técnica, Prieto et al. (2015) demonstraram a dificuldade na identificação da frequência dominante em séries temporais de $\mathrm{PM}_{10}$, sendo necessária, para contornar tal situação, a formulação de hipóteses simplificadoras que, por sua vez, adicionam erros à análise.

Assim, a análise de caos surge como ferramenta alternativa no estudo da dispersão de poluentes. São fenômenos elegíveis à metodologia caótica àqueles aperiódicos, sensíveis às condições iniciais, dissipativos, não lineares e determinísticos (SAVI, 2004). Em face deste cenário, o presente trabalho objetiva avaliar a evolução temporal de PM10 e $\mathrm{CO}$ à luz da teoria do caos determinístico para identificar as características não-lineares destes fenômenos. Os dados utilizados referem-se aos anos de 1998 a 2014, obtidos na Estação de Monitoramento da CETESB localizada no Parque Ibirapuera na cidade de São Paulo. A metodologia consistiu em avaliar os invariantes caóticos entropia de Kolmogorov e dimensão de correlação advindos das características topológicas dos atratores estranhos.

\subsection{Atratores estranhos}

Vander Stapen (1996) denomina como atrator a figura única formada a partir das linhas de evolução (e/ou trajetórias) de um determinado sistema após tempo infinito. De posse do atrator, é possível determinar, por meio de suas características topológicas, o estado em que se encontra o sistema em estudo, caracterizando um evento dinâmico (BRIONGOS e SOLER, 2004). Para um fenômeno caótico, sua solução está inserida em um espaço de n-dimensões, com linhas de fluxo aperiódicas que jamais se cruzam e nunca retornam a um estado anterior. 
Para séries temporais experimentais, Broomhead e King (1986) desenvolveram uma metodologia para a reconstrução do atrator no espaço de fases que utiliza as propriedades da matriz de covariância para esboçar o grau de independência de cada variável, obtendo variáveis não relacionadas. Tal método ficou conhecido como decomposição em valores singulares (SVD) e, a partir da seleção e separação das frequências características dos ruídos da série original, permite a reconstrução de um atrator representativo da dinâmica real do fenômeno.

\subsection{Entropia de Kolmogorov}

A entropia de Kolmogorov (K) solidificou-se como um dos procedimentos matemáticos mais utilizados na caracterização de processos caóticos. Análogo ao conceito termodinâmico clássico, a entropia de Kolmogorov também quantifica o grau de desorganização ou imprevisibilidade de um sistema, sendo para $K>0$ o sistema é considerado caótico; $\mathrm{K} \rightarrow \infty$ para sistemas estocásticos e $\mathrm{K}=0$ para sistemas determinísticos. A sua dinâmica, todavia, baseia-se na quantificação da taxa de perda de informações ao longo do atrator ou na previsibilidade de rota de certo ponto arbitrário (CASTILHO, 2011). Um método para estimar K é aproximá-lo à entropia de Verossimelhança $\left(\mathrm{K}_{\mathrm{ML}}\right)$ (SCHOUTEN et al., 1994), em que:

$$
K \approx K_{M L}=\frac{1}{\tau_{S}} \ln \left(1-\frac{1}{b}\right)
$$

O passo de tempo $\left(\tau_{\mathrm{S}}\right)$ é obtido pela frequência de amostragem $\left(\mathrm{f}_{\mathrm{S}}\right)$ como $\tau_{\mathrm{S}}=1 / \mathrm{f}_{\mathrm{S}}$ e o parâmetro b é definido como o número de cubos $\left(\mathrm{i}_{1}, \mathrm{i}_{2}, \mathrm{i}_{3}, \ldots, \mathrm{i}_{\mathrm{b}}\right)$ necessários para recobrir o atrator reconstruído no espaço de fases. Este método é largamente utilizado no cálculo da entropia de Kolmogorov para sinais experimentais, pois exibe uma menor influência de ruídos.

\subsection{Dimensão de correlação}

Vander Stapen (1996) define a dimensão de correlação como a medida da complexidade global do sistema por meio da quantificação do grau de heterogeneidade do atrator. Uma das metodologias clássicas de obtenção da dimensão de correlação $\left(D_{2}\right)$ utiliza-se do algoritmo proposto por Grassberger e Procaccia (1983). Neste método, a probabilidade de que dois pontos do atrator estejam inseridos em um hipercubo de lado $\omega$ é aproximada pela probabilidade de que estes mesmos pontos estejam espaçados a uma distância menor ou igual a $\omega$, sendo assim:

$$
D_{2}=\lim _{\omega \rightarrow 0} \frac{\log C(\omega)}{\log (\omega)}
$$

em que $C(\omega)$ é uma função de correlação da forma:

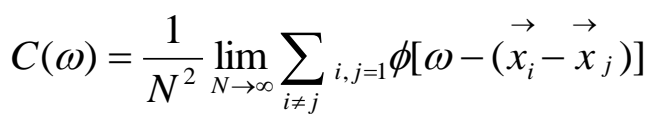

Nesta função, $\mathrm{N}$ é o número de pontos do atrator, $\vec{x}_{i}$ é o vetor associado à função de Heaviside $(\phi=1$ se $\mathrm{x} \geq 1$ ou $\phi=0$ se $\mathrm{x}<0) \mathrm{e}$ $\omega$ é comprimento do lado dos hipercubos que recobrem os pontos do atrator.

\section{MATERIAIS E MÉTODOS}

As séries temporais de concentração de material particulado inalável e $\mathrm{CO}$ foram diretamente exportadas da plataforma eletrônica da Companhia de Tecnologia de Saneamento Ambiental do Estado de SP (CETESB). Todas as análises foram realizadas no âmbito dos últimos 16 anos, ou seja, de 1998 a 2014; período máximo disponibilizado pela referida plataforma. A 
frequência de amostragem é de 1 hora, sendo 8.760 o número total de pontos de cada série.

O algoritmo utilizado para a reconstrução dos atratores, cálculo da entropia de Kolmogorov e dimensão de correlação foi o Basic Resolution of Chaos (BRChaos), implementado por Moura (2013). Os métodos encontrados no BRChaos são a decomposição em valores singulares (SVD) (BROOMHEAD e KING, 1986), máxima verossimilhança (SCHOUTEN et al., 1994) e de Grassberger e Procaccia (1983), respectivamente para a obtenção dos atratores, entropia de Kolmogorov e dimensão de correlação.

\section{RESULTADOS E DISCUSSÕES}

A identificação da dinâmica caótica em um determinado sistema não é tarefa trivial, sendo necessário lançar mão de um conjunto de ferramentas matemáticas que permitam avaliar o grau de imprevisibilidade e complexidade do processo. Após a obtenção de uma série temporal reconstrói-se, em um espaço de fases, o atrator e de suas características topológicas abstrai-se a dimensão de correlação e a entropia de Kolmogorov, invariantes característicos que determinam o quão caótica é a série avaliada. No que se refere aos fenômenos caóticos, estes apresentam, durante sua evolução temporal, uma contração no volume do espaço de fases e suas linhas de fluxo dependerão das condições iniciais (RUELLE e TAKENS, 1971) definindo o chamado atrator estranho ou atrator caótico. As Figuras 1a e $1 \mathrm{~b}$ apresentam as séries temporais e os atratores da concentração de PM $_{10}$ e CO para os anos de 1998, 2006 e 2013. A reconstrução dos atratores no espaço de fases foi realizada a partir das respectivas séries temporais com 8.760 pontos, dimensão de imersão (n) igual 3 e tempo de atraso $(\theta)$ de 1 hora. Para todas as figuras reconstruídas, nota-se que as linhas de fluxo, para as concentrações anuais de $\mathrm{PM}_{10} \mathrm{e}$ $\mathrm{CO}$, resultam em atratores estranhos, convergindo para uma região bem definida e estreita no espaço de fases (linha horizontal em $\mathrm{PC}_{2}$ (principal componente) $=0$ ), sugerindo uma dinâmica caótica determinista.

Devido às disformidades presentes no espaço de fases reconstruído, é importante que se avalie o grau de heterogeneidade do atrator para explicitar a complexidade global do sistema. Atualmente a grandeza mais utilizada para este fim é a dimensão de correlação $\left(D_{2}\right)$ e justifica-se esta escolha por dois motivos: o primeiro se dá pela dificuldade para resolver numericamente as dimensões fractais, pois a metodologia que permite sua obtenção baseia-se no algoritmo de contagem de caixas que, por sua vez, necessita de grande esforço computacional. Outro fator preponderante é que, uma vez calculada $\mathrm{D}_{2}$, pode-se obter o número de variáveis necessárias para descrever o comportamento caótico (MONTEIRO, 2011).

Para a obtenção de $\mathrm{D}_{2}$, por meio da aplicação da metodologia de Grassberger e Procaccia (1983), é necessário o conhecimento do tempo de atraso $(\theta)$. Com este fim, vários estudos versam sobre a melhor metodologia de determinação deste parâmetro. Alguns sugerem a utilização da informação mútua (FRASER e SWINNEY, 1986), função de auto correlação (HOLZFUSS e MAYER-KRESS, 1985), integral de correlação (LIEBERT e SCHUSTER, 1989; ISLAM et al., 1992; RAGA e Le MOYNE, 1996; YU et al., 2011; YU et al., 2013). Na prática, tem-se utilizado o tempo de atraso igual a frequência de dados (SUGIHARA e MAY, 1990;. HSIEH et al., 2008; SIVAKUMAR et al., 2001; GLASER et al., 2011; YU et al., 2011; YU et al., 2013). Por via de consequência, nos presente assume-se $\theta=1$ hora. 
Figura 1 - Séries temporais e diagramas de fase reconstruídos para concentrações anuais de:

(a) $\mathrm{PM}_{10}$; (b) $\mathrm{CO}$.

(a) $\mathrm{PM}_{10}$

1998
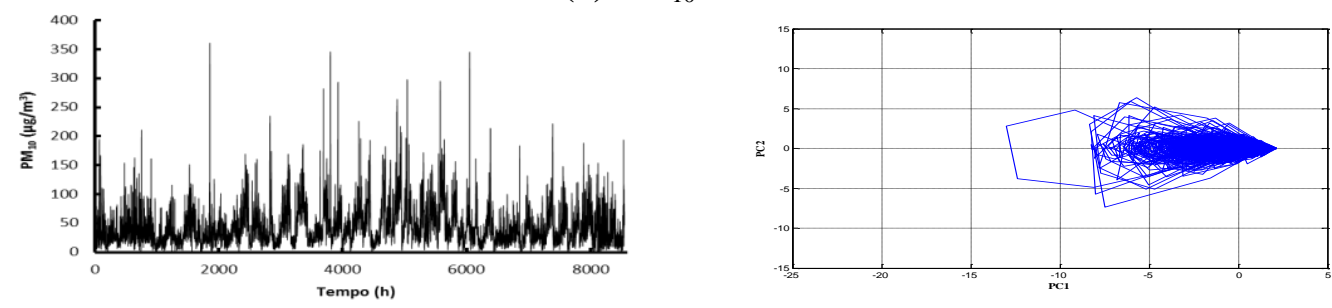

2006
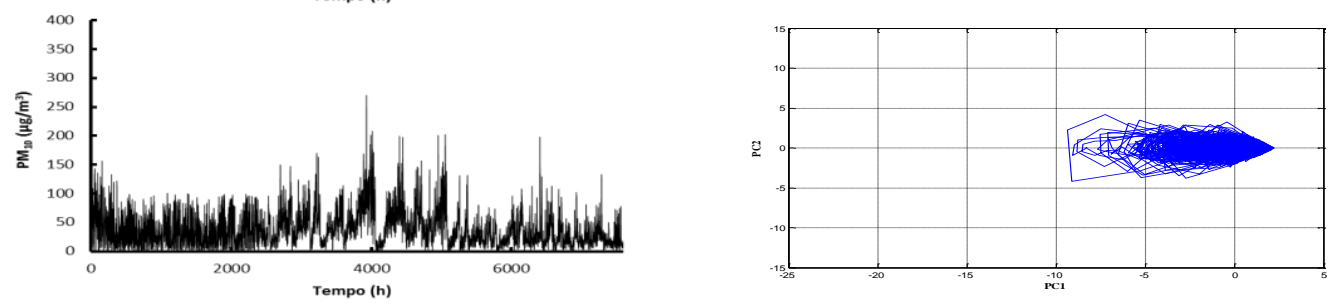

2013
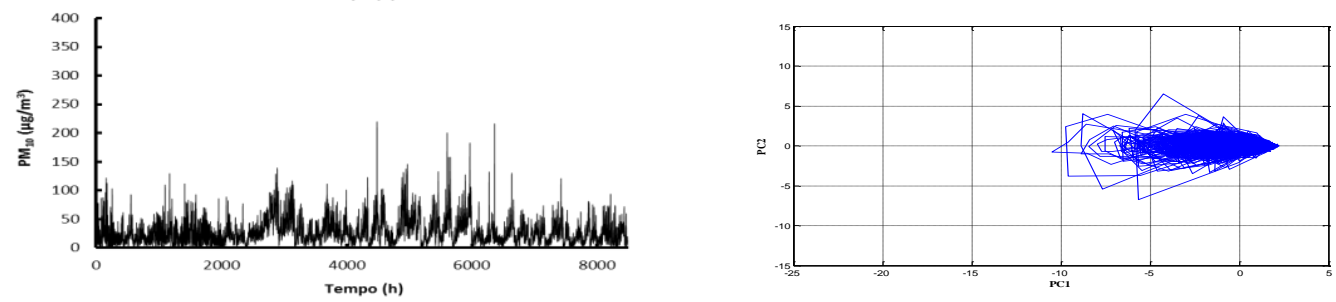

(b) $\mathrm{CO}$

1998
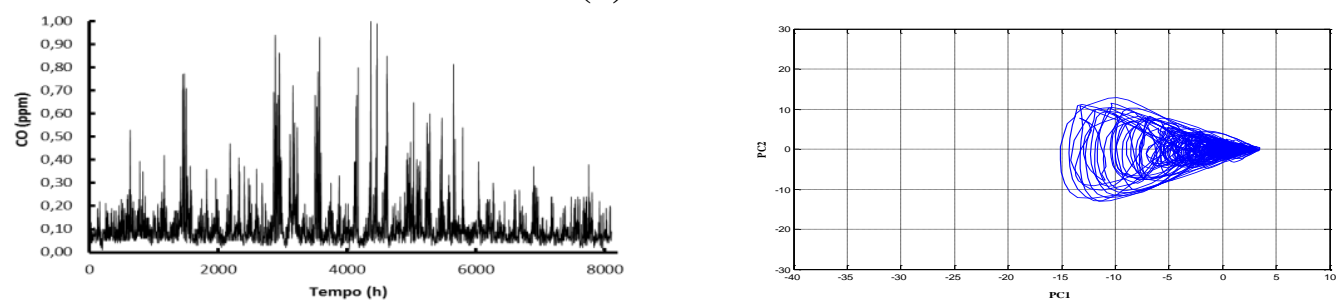

2006
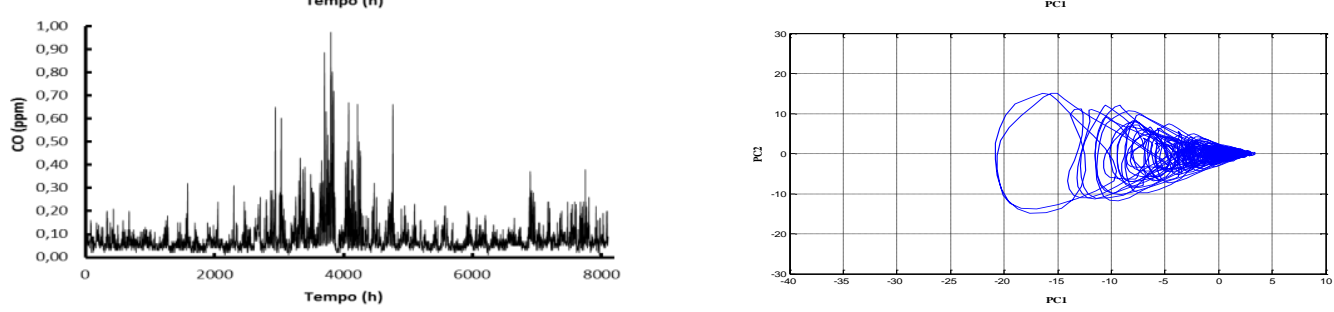

2013
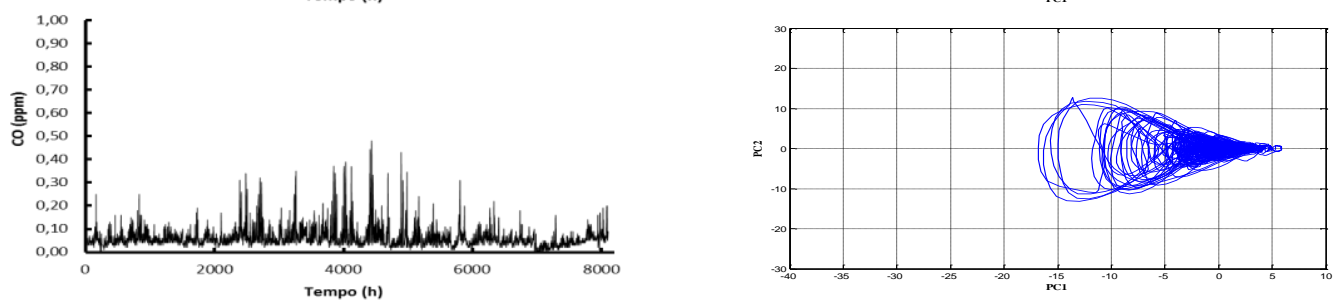

Para exemplificar, a Figura 2 apresenta a relação da integral de correlação $(C(\omega))$ com o raio $(\omega)$ ) e a convergência de $D_{2}$ com o

aumento de $n$ para as concentrações de $\mathrm{PM}_{10}$ (a) e CO (b) para o ano de 2006 para dimensões de imersão de 1 a 36. 
Figura 2 - Convergência da dimensão de correlação para concentrações anuais de:

(a) $\mathrm{PM}_{10}$; (b) CO.

(a) $\mathrm{PM}_{10}$
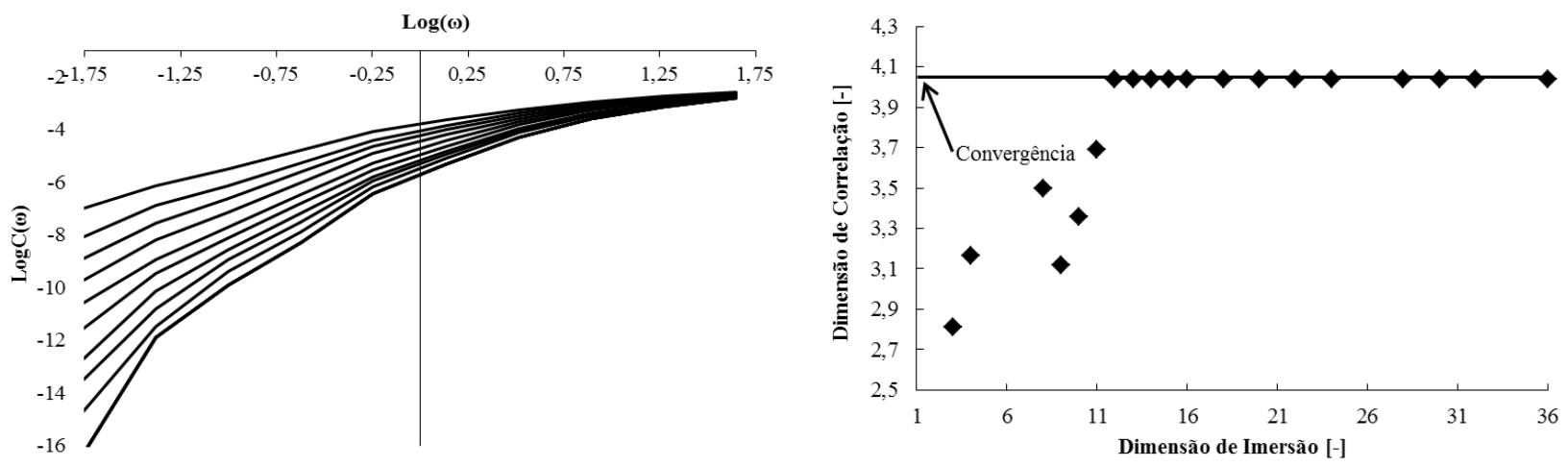

(b) $\mathrm{CO}$
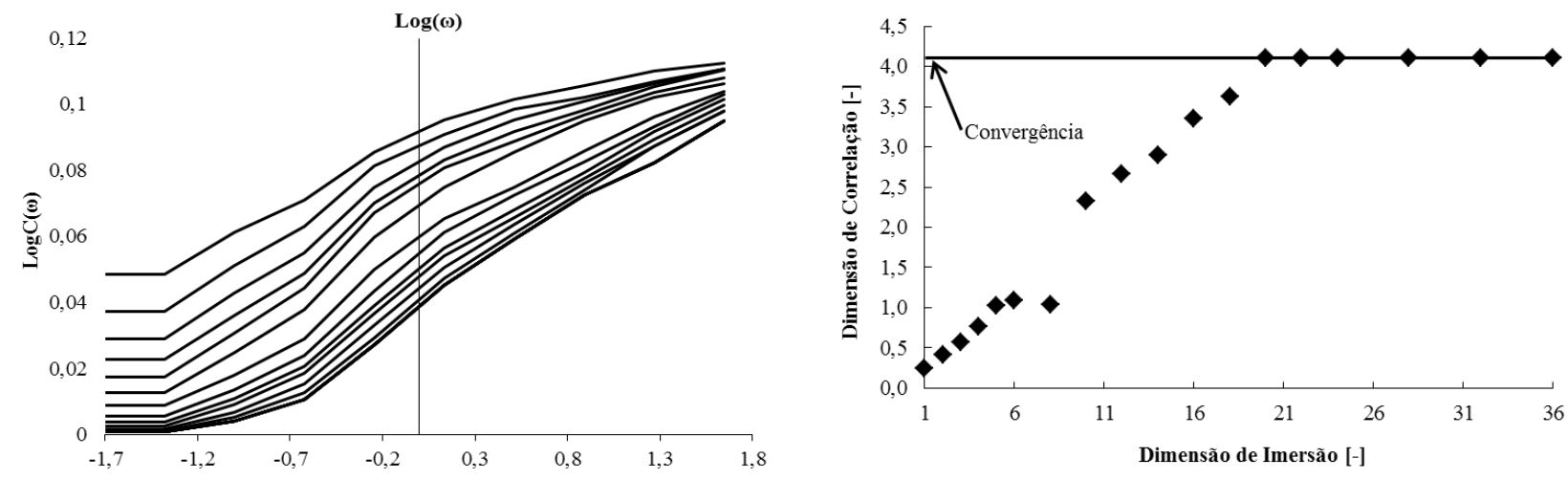

Tabela 1 - Dimensão de correlação e graus de liberdade das séries temporais de $\mathrm{PM}_{10}$ e CO .

\begin{tabular}{cccc|ccc}
\hline & \multicolumn{3}{c}{$\mathbf{P M}_{\mathbf{1 0}}$} & & \multicolumn{3}{c}{ CO } \\
\hline \multirow{2}{*}{ Ano } & $\begin{array}{c}\boldsymbol{n} \text { de } \\
\text { convergência }\end{array}$ & $\begin{array}{c}\mathbf{D}_{\mathbf{2}} \\
{[-]}\end{array}$ & Variáveis & $\begin{array}{c}\text { n de } \\
\text { convergência }\end{array}$ & $\begin{array}{c}\mathbf{D}_{\mathbf{2}} \\
{[-]}\end{array}$ & Variáveis \\
\hline $\mathbf{1 9 9 8}$ & 14 & 3,15 & 4 & 18 & 4,34 & 5 \\
$\mathbf{1 9 9 9}$ & 12 & 4,40 & 5 & 19 & 4,32 & 5 \\
$\mathbf{2 0 0 0}$ & 13 & 3,34 & 4 & 17 & 4,53 & 5 \\
$\mathbf{2 0 0 1}$ & 12 & 3,95 & 4 & 19 & 4,74 & 5 \\
$\mathbf{2 0 0 2}$ & 12 & 3,43 & 4 & 18 & 3,86 & 4 \\
$\mathbf{2 0 0 3}$ & 09 & 4,21 & 5 & 17 & 3,82 & 4 \\
$\mathbf{2 0 0 4}$ & 10 & 3,63 & 4 & 21 & 3,22 & 4 \\
$\mathbf{2 0 0 5}$ & 11 & 3,93 & 4 & 17 & 4,77 & 5 \\
$\mathbf{2 0 0 6}$ & 11 & 4,04 & 5 & 20 & 4,11 & 5 \\
$\mathbf{2 0 0 7}$ & 16 & 3,48 & 4 & 18 & 3,68 & 4 \\
$\mathbf{2 0 0 8}$ & 17 & 3,62 & 4 & 16 & 2,94 & 3 \\
$\mathbf{2 0 0 9}$ & 14 & 3,85 & 4 & 16 & 2,89 & 3 \\
$\mathbf{2 0 1 0}$ & 18 & 4,17 & 5 & 19 & 3,68 & 4 \\
$\mathbf{2 0 1 1}$ & 16 & 3,57 & 4 & 17 & 2,91 & 3 \\
$\mathbf{2 0 1 2}$ & 14 & 3,90 & 4 & 16 & 3,17 & 4 \\
$\mathbf{2 0 1 3}$ & 12 & 4,04 & 5 & 18 & 3,31 & 4 \\
$\mathbf{2 0 1 4}$ & - & - & - & 21 & 2,88 & 3 \\
\hline
\end{tabular}


Nas séries temporais obtidas, o valor da dimensão de correlação cresce até um ponto em que a taxa de aumento começa a diminuir e os valores de $\mathrm{D}_{2}$ atingem a saturação. Este ponto de saturação aponta, precisamente, a presença de uma dinâmica determinista (YU et al., 2013). Além disso, na evolução da dimensão de correlação, baixas dimensões são indicativos da formação de atratores do tipo ponto fixo no diagrama de fases. Após este ponto, ocorre formação de estruturas do tipo ciclo limite e o aumento de $\mathrm{D}_{2}$ indica a presença de instabilidades causadoras de duplicação de período, resultando em dinâmica caótica para as dimensões maiores que 2 e 3. Para as situações apresentadas na Figura 2, a saturação e, portanto, o caos determinístico, é atingida para $\mathrm{D}_{2}=4,04 \mathrm{e}$ 3,31 , respectivamente, para as concentrações de $\mathrm{PM}_{10}$ e CO. Via de regra, adota-se o maior número inteiro mais próximo como a quantidade de variáveis que regem a dinâmica do sistema (YU et al., 2013). A Tabela 1 traz o cálculo da dimensão de correlação, $n$ de convergência e número de variáveis para as concentrações anuais de $\mathrm{PM}_{10}$ e $\mathrm{CO}$ nos intervalos de 1998 a 2014. Observa-se que para os dois poluentes os números de variáveis do sistema apresentam valores anuais diferentes, ou seja, não há unicidade no que diz respeito à dinâmica dos sistemas avaliados.

Em termos de fenômeno, as séries temporais de concentração de poluentes atmosféricos são diretamente afetadas por fatores naturais e antropogênicos que variam constantemente tanto em frequência quanto em magnitude e impactam decisivamente na interação do poluente-ambiente e, portanto, sua mensuração, culminando em diferentes dinâmicas temporais, mais ou menos complexas, incrementando dificuldade na identificação destas forças motrizes e de suas contribuições presentes na série temporal (YU et al., 2013).
É importante mencionar que a série temporal de $\mathrm{PM}_{10}$ para o ano de 2014 apresentou diversos dados inválidos, ou seja, informações não computadas neste estudo, desta forma impactando e inviabilizando a avaliação do referido período.

Em última análise, lança-se mão da entropia de Kolmogorov para certificar a natureza caótica dos sistemas em estudo. $\mathrm{Na}$ Tabela 2 estão relacionados os valores de entropia de Kolmogorov das séries temporais analisadas. Em todas as situações verificou-se a presença de caos e, como já esperado, os valores anuais apresentam-se em mesma ordem de grandeza, porém diferentes. Esse resultado é corroborado pelo fato de que as séries temporais são definitivamente afetadas por fatores meteorológicos (frequência e volume de precipitações, velocidade do ar etc.), implementação de políticas públicas e alterações físicas e imprevisíveis na estação de monitoramento, conduzindo, assim, a situações anuais mais ou menos aleatórias.

Tabela 2 - Entropia de Kolmogorov das séries temporais de $\mathrm{PM}_{10}$ e $\mathrm{CO}$.

\begin{tabular}{ccc} 
& PM $_{\mathbf{1 0}}$ & CO \\
\hline Ano & $\begin{array}{c}\text { K } \\
\text { (bits/s) }\end{array}$ & $\begin{array}{c}\text { K } \\
\text { (bits/s) }\end{array}$ \\
\hline $\mathbf{1 9 9 8}$ & 0,1944 & 0,1214 \\
$\mathbf{1 9 9 9}$ & 0,2283 & 0,0974 \\
$\mathbf{2 0 0 0}$ & 0,2211 & 0,1078 \\
$\mathbf{2 0 0 1}$ & 0,2943 & 0,1154 \\
$\mathbf{2 0 0 2}$ & 0,2771 & 0,0995 \\
$\mathbf{2 0 0 3}$ & 0,2666 & 0,1048 \\
$\mathbf{2 0 0 4}$ & 0,3080 & 0,1172 \\
$\mathbf{2 0 0 5}$ & 0,3435 & 0,1084 \\
$\mathbf{2 0 0 6}$ & 0,2326 & 0,0959 \\
$\mathbf{2 0 0 7}$ & 0,1549 & 0,1084 \\
$\mathbf{2 0 0 8}$ & 0,1555 & 0,1079 \\
$\mathbf{2 0 0 9}$ & 0,2302 & 0,1516 \\
$\mathbf{2 0 1 0}$ & 0,1185 & 0,1167 \\
$\mathbf{2 0 1 1}$ & 0,1909 & 0,1077 \\
$\mathbf{2 0 1 2}$ & 0,2015 & 0,1361 \\
$\mathbf{2 0 1 3}$ & 0,2179 & 0,1340 \\
$\mathbf{2 0 1 4}$ & - & 0,1219 \\
\hline
\end{tabular}


Em linhas gerais, os parâmetros meteorológicos influenciam diretamente a amplitude da evolução temporal de concentração de $\mathrm{PM}_{10}$ e $\mathrm{CO}$, porém qualquer alteração na região onde está instalada a estação também afeta as séries temporais, podendo torná-las mais ou menos previsíveis, desta forma influenciando os invariantes caóticos. Algumas possíveis mudanças na região da estação que se podem influenciar os resultados são: o aumento ou diminuição de fontes poluentes (veículos e indústrias); deslocamento de pessoas e veículos (causando suspensão de, por exemplo, material particulado depositado no solo); aumento ou diminuição do número de árvores etc. Neste sentido, a estação do Ibirapuera situa-se em um ponto atípico da região metropolitana da cidade de São Paulo, pois se trata de uma região residencial, bem arborizada, não apresentando fontes industriais em seu entorno; sendo as fontes veiculares as responsáveis pelas emissões locais. No caso dos $\mathrm{PM}_{10}$, o deslocamento de pessoas e veículos também aumenta as concentrações de particulados na região. $\mathrm{O}$ aumento do número de veículos é o fator que mais impactou longo dos anos, porém políticas de fiscalização e controle de emissões veiculares amorteceram parte desse impacto.

No que diz respeito à análise de caos, Yu et al (2011) e Yu et al (2013) concluem que esta metodologia é adequada para avaliar séries temporais de poluentes atmosféricos, pois por meio dela, mais especificamente do cálculo da dimensão de correlação, é possível obter resultados muito próximos daqueles postulados por análises clássicas como DFA, porém o caos determinístico considera de forma ampla as relações não lineares associadas ao fenômeno com a vantagem de custo computacional muito menor daquele exigido por metodologias tradicionais.

\section{CONCLUSÕES}

A avaliação inicial da presença do caos determinístico em séries temporais de concentração anual de $\mathrm{PM}_{10}$ e $\mathrm{CO}$ na estação de monitoramento (CETESB) do Ibirapuera, São Paulo, demonstrou a natureza caótica dos sistemas, deixando claro que a poluição atmosférica apresenta, durante sua evolução temporal, atratores do tipo estranhos e, intuitivamente, uma dinâmica dissipativa, não linear, aperiódica a longo prazo, determinística e extremamente sensível às condições iniciais.

Por meio da aplicação do método da integral de correlação foi possível obter o número de variáveis que regem a dinâmica da poluição atmosférica para os dois parâmetros avaliados, explicitando a presença de caos nos dados estudados.

A metodologia empregada neste trabalho descreve adequadamente, no que se refere aos poluentes considerados, a dinâmica da poluição do ar, indicando a sua possível aplicação no entendimento dos padrões, mecanismos e causas deste fenômeno.

\section{REFERÊNCIAS}

AMDUR, M. O; DOULL, J.; KLAASSEN, C. D. Casarett and Doull's toxicology: The basic science of poisons. 4th ed. Oxford: Pergamon Press; 1991.

BAI, D.; GRACE, J.R.; ZHU, J.X.; Characterization of gas fluidized beds of group $\mathrm{C}, \mathrm{A}$ and $\mathrm{B}$ particles based on pressure fluctuations, Canadian Journal of Chemical Engineering, 77, p. 319, 1999.

BRAGA, A. L. F.; CONCEIÇÃO, G. M. S.; PEREIRA, L. A. A.; Kishi H.; PEREIRA, J. C. R.; ANDRADE, M. F.; et al. Air pollution and pediatric respiratory hospital admissions in Sao Paulo, Brazil. J Environ Med. 1999; 1:95-102. 
BROOMHEAD, D. S.; KING, G. P. Extracting qualitative dynamics from experimental data. Physica 20 D, pp. 217-236, 1986.

BROWN, R. C.; BRUE, E.; Resolving dynamical features of fluidized beds from pressure fluctuations, Powder Technology, 119, p. 68, 2001.

CASTILHO, G. J. Análise de Caos em Leito Fluidizado Circulante. Tese (Doutorado em Engenharia Química), Faculdade de Engenharia Química, Universidade Estadual de Campinas, Campinas-SP, 2011.

DERISIO, J. C. Introdução ao controle de poluição ambiental. São Paulo: CETESB; 1992.

DOCKERY, D. W.; POPE III, C. A. Acute respiratory effects of particulate air pollution. Annu Rev Public Health 1994;15:107-32.

DUCHIADE, M. P. Poluição do ar e doenças respiratórias: uma revisão. Cad. Saúde Pública, Set 1992, vol.8, no.3, p.311-330.

FRASER, A. M.; SWINNEY, H. L. Independent coordinates for strange attractors from mutual information. Phys. Rev. A 33, 1134-1140. 1986.

GLASER, S. M..; YE, H.; MAUNDER, M..; MACCALL, A.; FOGARTY, M.;; SUGIHARA, G. 2011. Detecting and forecasting complex nonlinear dynamics in spatially structured catch-per-unit-effort time series for North Pacific albacore (Thunnus alalunga). Can. J. Fish. Aquat. Sci. 68, 400 - 412.

GRASSBERGER, P.; PROCACCIA, I. Measuring the Strangeness of Strange Attractors. In Physica D, v.9, 189-208, 1983

GUO, H., 2011. Source apportionment of volatile organic compounds in Hong Kong homes. Build Environ. 46, 2280 a 2286.

HELLEBUST, S.; ALLANIC, A.; O'CONNOR, I. P.; WENGER, J. C.; SODEAU, J. R., 2010. The use of real-time monitoring data to evaluate major sources of airborne particulate matter. Atmos. Environ. 44, $1116-1125$.

HOLZFUSS, J.; MAYER-KRESS, G. Approach to Error-estimation in the Application of Dimension Algorithms. Los Alamos National Lab, NM (USA). 1985.

HONG, S. C.; JO, B. R.; DOH, D. S.; CHOI, C. S. Determination of minimum fluidization velocity by the statistical analysis of pressure fluctuations in a gas-solid fluidized bed, Powder Technology, 60, p. 215, 1990.

HSIEH, C.H.; ANDERSON, C.; SUGIHARA, G. Extending Nonlinear analysis to short ecological time series. Am. Nat. 171, 71 - 80, 2008

ISLAM, S.; CHEN, J. L.; BISWAS, P. Nonlinear dynamics of hourly ozone concentrations: nonparametric short term prediction. Atmos. Environ. 32, 1839-1848, 1998.

JOHNSSON, F.; ZIJERVELD, R. C.; SCHOUTEN, J. C.; VAN DEN BLEEK, C. M., LECKNER, B. Characterization of fluidization regimes by time-series analysis of pressure fluctuations. International Journal of Multiphase Flow, v. 26, p. 663-715, 2000.

KAGE, H.; IWASAKI, N.; YAMAGUCHI, H.; MATSUNO, Y. Frequency analysis of pressure fluctuation in fluidized bed plenum. Journal of Chemical Engineering of Japan, 24, p. $76-81$, 1991.

LIEBERT, W.; SCHUSTER, H. G., 1989. Proper choice of the time-delay for the analysis of chaotic time-series. Phys. Lett. A 142, 107-111.

LORENZ, E. N. Deterministic nonperiodic flow. J. Atmos. Sci., v. 20, pp. 130-141, 1963.

MARTIN, A. E.; BRADLEY, W. H. Mortality, fog, and atmospheric pollution: an investigation during the winter of 1958-59. Month Bull Min Hlth. 19: 56. 1960 
MARTÍN, L.; BRIONGOS, J. V.; HERNANDO, G. N.; ARAGÓN, J. M. Detecting regime transitions in gas-solid fluidized beds from low frequency accelerometry signals, Powder Technology, 207, p. $104-112,2011$.

MONTEIRO, L. H. A. Sistemas Dinâmicos. $2^{\mathrm{a}}$ Ed. São Paulo: Livraria da Física, 2011.

MOURA, H. L. Análise da influência do ruído na obtenção de atratores e parâmetros determinísticos da teoria do caos aplicada em uma seção de riser de um leito fluidizado circulante. Faculdade de Engenharia Química, UNICAMP, Campinas-SP, 2013.

POPE, C. A. I.; BURNETT, R. T.; THUN, M. J.; CALLE, E. E.; KREWSKI, D.; ITO, K.; THURSTON, G. D. Lung cancer, cardiopulmonary mortality, and long-term exposure to fine particulate air pollution. J. Am. Med. Assoc. 287, 1132-1141. 2002

PRIETO, W. H.; TOMAZ, E.; CREMASCO, M. A.; Aplicação da Teoria do Caos em dados de concentração de material particulado inalável na atmosfera, p. 8540-8547. In: Anais do XX Congresso Brasileiro de Engenharia Química - COBEQ 2014 [= Blucher Chemical Engineering Proceedings, v.1, n.2]. São Paulo: Blucher, 2015.

RAGA, G. B.; LE MOYNE, L. On the nature of air pollution dynamics in Mexico City. 1. Nonlinear analysis. Atmos. Environ. 30, 3987 - 3993. 1996.

Relatório de Qualidade do Ar no Estado de São Paulo - 2013, Companhia de Tecnologia de Saneamento Ambiental-CETESB, 2014.

RUELLE, D.; TAKENS, F. On the nature of turbulence, Commum. Math. Physics, v. 30, pp. 167192, 1971.

SALDIVA, P. H. N.; POPE III, C. A.; SCHWARTZ, J.; DOCKERY, D. W.; LICHTENFELS, A. J.; SALGE, J. M.; et al. Air pollution and mortality in elderly people: a time-series study in Sao Paulo, Brazil. Arch Environ Health; 50:159-63. 1995.

SASIC, S.; LECKNER, B.; JOHNSSON, F. Characterization of fluid dynamics of fluidized beds by analysis of pressure fluctuations, Progress in Energy and Combustion Science, 33, p. 453-496, 2007.

SCHOUTEN, J. C.; TAKENS, F.; VAN DEN BLEEK, C. M. Maximum-Likelihood-Estimation of the Entropy of an Attractor. In Physical Review E, v. 49, pp. 126-129, 1994.

SIVAKUMAR, B.; SOROOSHIAN, S.; GUPTA, H. V.; Gao, X. G. A chaotic approach to rainfall disaggregation. Water Resour. Res. 37, 61-72. 2001.

SUGIHARA, G., MAY, R. M.. Nonlinear forecasting as a way of distinguishing chaos from measurement error in time-series. Nature 344, 734 - 741. 1990.

TRNKA, O.; VESELÝ, V.; HARTMAN, M.; BERAN, Z. Identification of the state of a fluidized bed by pressure fluctuation, AIChE Journal, 46, p. 509, 2000.

TSONIS, A. A. Chaos: From Theory to Applications. New York: Plenum Press, 274 p. 1992.

VANDER STAPPEN, M. L. Chaotic hydrodynamics of fluidized beds, Delft: University of Technology, Delft-NL, 236 pp. PhD. Thesis, 1996.

YU, B.; HUANG, C.; LIU, Z; WANG, H. WANG, L. A chaotic analysis on air pollution index change over past 10 years in Lanzhou, northwest China. Stoch Environ Res Risk Assess (2011) 25:643-653

YU, H-L.; LIN, Y-C.; SIVAKUMAR, B.; KUO, YM. A study of the temporal dynamics of ambient particulate matter using stochastic and chaotic techniques. Atmospheric Environment 69, 37-45, 2013. 